\title{
Epileptischer Krampfanfall - Das sollten Sie wissen für die Ergänzungsprüfung
}

Rico Kuhnke, Thomas Ahne

retten! macht Sie fit für den Notfallsanitäter: In jeder Ausgabe arbeiten wir anhand eines Fallbeispiels einen interessanten Einsatz algorithmenkonform auf. Anhand von exemplarischen Fragen zu erweiterten Notfallmaßnahmen, Kommunikation und Rahmenbedingungen können Sie sich auf die Ergänzungsprüfung vorbereiten - egal, in welchem Bundesland Sie arbeiten.

\section{Fallbeispiel}

\section{Einsatzmeldung}

An einem Dienstagvormittag gegen 10:00 Uhr wird die Besatzung des Rettungswagens in einen Elektromarkt zu einer bewusstlosen Person geschickt. Die Verkäuferin erzählt, ein junger Mann liege zwischen den Regalen ohnmächtig am Boden. Sie selbst habe den Mann nicht gesehen; sie sei nur vom Marktleiter angewiesen worden, den Rettungsdienst zu dem Betroffenen zu bringen. Vor dem Eingang wartet eine Verkäuferin auf das Team. Das Team entscheidet, das Material (Notfallrucksack, EKG, Absaugeinheit, Beatmungsgerät) auf der Fahrtrage zu belassen.

\section{Situation vor Ort}

Ein junger Mann liegt regungslos zwischen den Regalen. Neben ihm kniet eine jüngere Frau, die dem Mann ein Taschentuch auf eine Wunde an der Stirn drückt. Neben den beiden steht der Marktleiter; interessierte Kunden bittet er, weiterzugehen.

Die Frau erzählt, sie sei die Betreuerin des jungen Mannes. Er sei bekannter Epileptiker und habe eine mäßige 
geistige Behinderung. $\mathrm{Er}$ arbeite in einer Behindertenwerkstatt in der Nähe. Alexander habe sich ein neues Mobiltelefon kaufen wollen, und sie habe ihn zu dem Einkauf begleitet, als er plötzlich und unerwartet zu Boden gestürzt sei. Dabei habe er sich wohl die Stirn gestoßen und verletzt. Am Boden habe er dann einen generalisierten Krampfanfall mit tonisch-klonischen Krämpfen gehabt. Dieser habe ca. 2 Minuten angehalten.

\section{Erneuter Krampfanfall}

Noch bevor sich das Team um die Versorgung kümmern kann, beginnt der junge Mann erneut zu krampfen. Die Betreuerin hält vorsichtig den Kopf des Patienten. Wegen der heftigen tonisch-klonischen Krämpfe an beiden Armen und Beinen entschließt sich das Team zur intranasalen Gabe von $10 \mathrm{mg}$ Midazolam. Eine Kollegin bereitet das Medikament vor, während die andere die Absaugbereitschaft herstellt und gemeinsam mit der Betreuerin darauf achtet, dass sich der Patient nicht verletzen kann.

Um eine versehentliche Überdosierung zu vermeiden, zieht die Kollegin nur $2 \mathrm{ml}$ des Medikaments auf eine Spritze auf, $1 \mathrm{ml}$ verbleibt in der Ampulle (3-ml-Ampulle $=15 \mathrm{mg}$ Midazolam). Der Patient erhält danach je $1 \mathrm{ml}$ der Lösung pro Nasenloch über einen Nasalzerstäuber (MAD). Nach einer knappen Minute beginnen die Krämpfe nachzulassen, kurz darauf verschwinden sie gänzlich.

\section{Postiktale Phase}

Nachdem der epileptische Krampfanfall zu Ende ist, können die beiden Kolleginnen sich um die weitere Versorgung des Patienten kümmern. Eine von ihnen versorgt die Kopfplatzwunde, die andere erhebt bei der Betreuerin eine Fremdanamnese. Dazu nutzt sie das SAMPLER-Schema.

\section{ZUSATZINFO}

Gängige Merkhilfen und Akronyme, vom ABCDESchema über das SAMPLER+S-Schema bis zu den $4 \mathrm{Hs}$ und HITS, haben wir für Sie übersichtlich zusammengefasst. Bestellen Sie Ihren kostenfreien Lernspicker zur Vorbereitung auf Ihre Ergänzungsprüfung unter www.thieme.de/retten-lernspicker.

\section{SAMPLER-Schema}

\section{S-ymptome}

Der Patient heißt Alexander und ist 21 Jahre alt. Er ist schläfrig und desorientiert. Auf Ansprache und leichtes Rütteln reagiert er abweisend.

\section{A-llergien}

Es sind keine Allergien bekannt.

\section{M-edikamente}

Alexander ist als Epileptiker mit Valproat $A b Z{ }^{\circledR} 500 \mathrm{mg}$ in der Kombination mit Levetiracetam UCB ${ }^{\circledR} 500$ mg eingestellt. Die genaue Dosierung ist der Betreuerin nicht bekannt. Bei einem Krampfereignis, das länger als 2 Minuten dauert, oder bei wiederkehrenden Krampfanfällen innerhalb einer Stunde soll der Patient 15 mg Dormicum (Midazolam) als Rectiole erhalten. Die Betreuerin gibt allerdings an, sie habe noch keine Gelegenheit zur Applikation gehabt. Sie zeigt auf ein Notfalltäschchen mit einer Tablettenbox und der Rectiole.

\section{P-atientengeschichte}

Bei dem Patienten ist eine erblich bedingte Epilepsie seit der frühesten Kindheit bekannt. Aufgrund einer geistigen Behinderung lebt er in einer Wohngruppe des nahegelegenen Epilepsiezentrums und arbeitet tagsüber in der angegliederten Behindertenwerkstatt. Er könne sich zwar grundsätzlich selbst versorgen, brauche allerdings Unterstützung bei Aufgaben des täglichen Lebens.

\section{L-etzte Mahlzeit, ...}

Das gemeinsame Frühstück heute Morgen sei gegen 8:00 Uhr gewesen. Danach habe er wohl nichts mehr gegessen. Sie sei sich da allerdings nicht sicher, da Alexander eine Vorliebe für Schokoriegel habe und immer mal wieder eine Zwischenmahlzeit zu sich nehme.

\section{E-reignis}

Sie habe Alexander beim Einkaufen begleitet. Plötzlich habe er aufgestöhnt und sei dann zu Boden gefallen. Dort habe er etwa 2 Minuten lang generalisierte tonischklonische Krämpfe gehabt.

\section{R-isikofaktoren}

Bekannte Epilepsie.

\section{ABCDE-Schema}

Während sich die Kollegin um die Fremdanamnese kümmert, verschafft sich ihre Teampartnerin einen kurzen Überblick über die Vitalwerte. Dazu nutzt sie das ABCDE-Schema.

A-irway

Die Atemwege sind frei. Die Mundinspektion zeigt einen lateralen Zungenbiss ( $\triangleright$ Abb. 1).

\section{B-reathing}

Die Atmung ist mit einer Frequenz von über 20 Atemzügen pro Minute schnell. Die Atemzüge sind regelmäßig und vertieft. Der Brustkorb hebt und senkt sich seitengleich. Beidseits ist ein unauffälliges vesikuläres Atemgeräusch zu hören. Die angelegte Pulsoxymetrie zeigt einen $\mathrm{SpO}_{2}$ von $96 \%$. Die Kollegin appliziert über eine Nasensonde 5 I Sauerstoff pro Minute. 


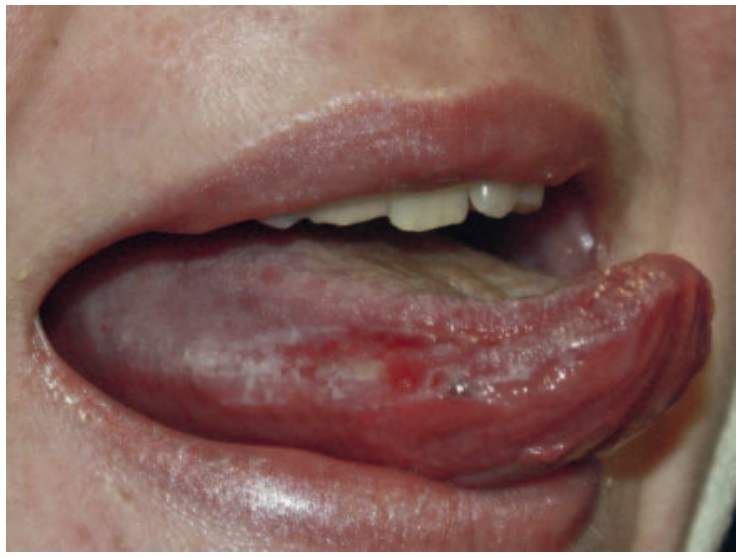

- Abb. 1 Zungenbiss (Quelle: Klinik für Epileptologie Bonn; aus: von Wrede R. Körperliche Untersuchung. In: Scholz J, Gräsner J, Bohn A, Hrsg. Referenz Notfallmedizin. 1. Auflage. Stuttgart: Thieme; 2019. doi:10.1055/b-006149615).

\section{C-irculation}

Der periphere Puls an der Ateria radialis ist gut tastbar, regelmäßig und mit einer Frequenz von 100 Schlägen pro Minute nur unwesentlich erhöht. Die Rekapillarisierungszeit des Nagelbetts liegt unter $2 \mathrm{~s}$. Der Blutdruck ist mit $140 / 70 \mathrm{mmHg}$ leicht erhöht, aber insgesamt unauffällig. Das angelegte EKG ist unauffällig.

\section{D-isability}

Alexander ist schläfrig und desorientiert. Auf Ansprache reagiert er ungehalten und abweisend. Er gibt an, dass er schlafen möchte. Die Pupillen des Patienten sind isokor und mittelweit, sie reagieren prompt auf Licht. Das Blutzuckertestgerät zeigt einen BZ von $130 \mathrm{mg} / \mathrm{dl}$. Die Temperatur ist mit $37,1^{\circ} \mathrm{C}$ normotherm.

\section{E-xposure}

Aufgrund der Situation im Supermarkt wird der Patient für eine Inspektion nicht entkleidet. Die Kollegin verschafft sich lediglich einen Überblick, achtet allerdings in besonderem Maß auf Hinweise für weitere Verletzungen. Außer einer ca. $2 \mathrm{~cm}$ langen Kopfplatzwunde über der rechten Augenbraue finden sich keine Verletzungen. Auffallend sind allerdings kleinere petechiale Einblutungen im Augenbereich ( $\triangleright$ Abb. 2).

Die Anwendung des FAST-Schemas (F-ace, A-rms, S-peach, T-ime) zum Ausschluss einer zerebralen Ischämie ist nur eingeschränkt möglich. Eine zerebrale Ischämie ist allerdings nicht wahrscheinlich. Bei der Ganzkörperinspektion fällt zudem auf, dass der Patient eingenässt hat.

\section{Transport in die Klinik}

Da der Patient immer noch desorientiert ist und die Kopfplatzwunde genäht werden muss, entscheidet sich das

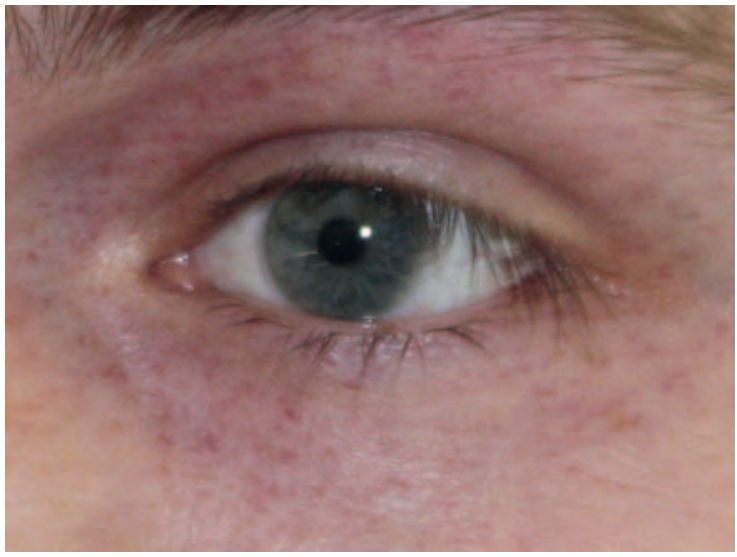

- Abb. 2 Periokuläre petechiale Einblutungen („Forellenaugen“) (Quelle: Klinik für Epileptologie Bonn; aus: von Wrede R. Körperliche Untersuchung. In: Scholz J, Gräsner J, Bohn A, Hrsg. Referenz Notfallmedizin. 1. Auflage. Stuttgart: Thieme; 2019. doi:10.1055/b-006-149615).

Team gemeinsam mit der Betreuerin für einen Transport in die Klinik. Alexander wird vorsichtig auf ein Tragetuch verbracht und danach auf die Fahrtrage übergehoben. Während des Transports wird er zunehmend wacher und orientierter. Die Anwesenheit der bekannten Betreuerin nimmt Alexander die Angst, und er lacht, als sie ihm seinen Kopfverband in einem Handspiegel zeigt.

\section{Der Algorithmus}

Das Team arbeitet in diesen Fall mit einem Musteralgorithmus des DBRD zur Versorgung eines Krampfanfalls bei Erwachsenen ( $\triangleright$ Abb.3). Die Musteralgorithmen orientieren sich am sogenannten Pyramidenprozess und werden regelmäßig überarbeitet. Dabei werden aktuelle Empfehlungen und Leitlinien mit einbezogen.

\footnotetext{
Merke

Laden Sie kostenfrei die Algorithmen des DBRD herunter unter www.dbrd.de.

Sollten Sie in Ihrem Rettungsdienstbereich einen abweichenden Algorithmus nutzen, arbeiten Sie die Unterschiede heraus und überlegen Sie sich, wie der Einsatz mit dem Ihnen zur Verfügung stehenden Algorithmus ablaufen müsste.
}

\section{Differenzialdiagnose Krampfanfall}

Bei Eintreffen des Rettungsdienstteams ist der Krampfanfall bereits zu Ende. Die Betreuerin gibt an, der Patient sei bekannter Epileptiker. Die Beschreibung des Krampfereignisses zusammen mit der Vorgeschichte des Patienten legt nahe, dass es sich bei dem geschilderten Ereignis um einen epileptischen Krampfanfall handelt. 


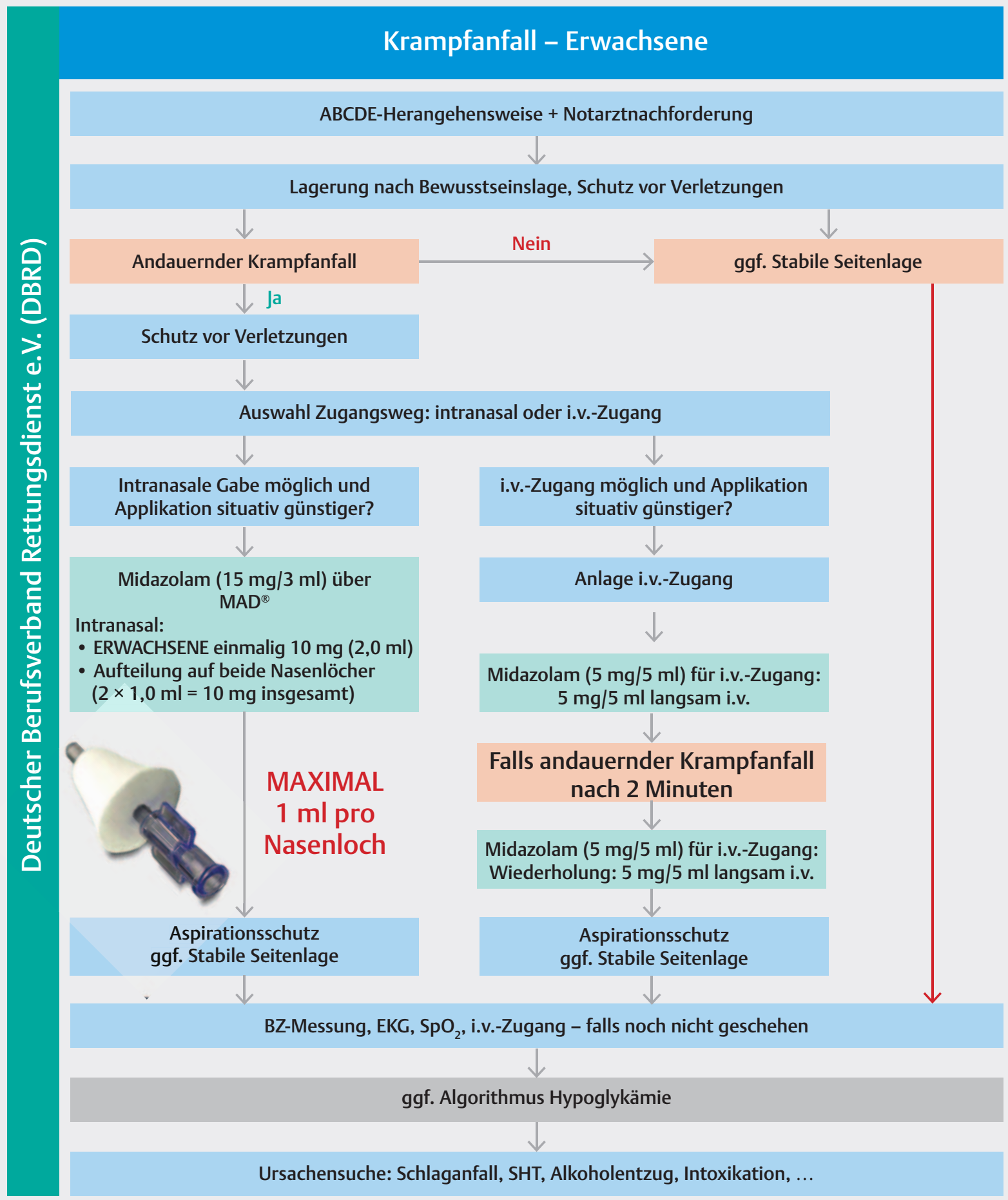

- Abb. 3 Algorithmus Krampfanfall - Erwachsene (Quelle: Deutscher Berufsverband Rettungsdienst e. V., DBRD).

Da der Krampfanfall nur Symptom und kein eigenständiges Krankheitsbild ist, ist eine gründliche Differenzialdiagnose sinnvoll. Dies gilt insbesondere dann, wenn das Krampfereignis erstmals auftritt. Typisch sind Krampfanfälle auch bei Schädel-Hirn-Traumen (SHT), im Rahmen einer Hypoglykämie oder anderer Stoffwechselentgleisungen, bei fieberhaften Infekten, intrazerebralen Blutungen bzw. zerebralen Ischämien und als Begleiterscheinung einer vasovagalen Synkope oder eines Entzugs. Zudem können auch psychogene Krampfanfälle auftreten. 


\section{Cave}

Bei einer Synkope kann es am Boden zu initialem Hochziehen der Arme und einzelnen Zuckungen kommen, die oft als epileptischer Krampf fehlgedeutet werden. Bei der Anamnese ist deshalb genau zu erfragen, wie sich der Anfall dargestellt hat: Was ging dem Krampf voraus? Waren es tonische und/oder klonische Krämpfe? Welche Muskelgruppen waren betroffen? Beidseitig oder einseitig? Beuge- und/oder Strecksynergismen? Wie lange hat das Ereignis gedauert?

\section{Midazolam intranasal}

Bevor das Team eine eingehende Untersuchung und Versorgung vornehmen kann, kommt es erneut zu einem Krampfanfall. Angesichts der generalisierten tonisch-klonischen Krämpfe ist das Legen eines i.v. Zugangs kaum möglich. Das Team entscheidet sich für die intranasale Applikation von $10 \mathrm{mg}$ Midazolam laut Musteralgorithmus.

Viele internationale Studien sowie diverse klinische Berichte aus Deutschland beschreiben einen schnellen Wirkungseintritt von Midazolam bei intranasaler Gabe. Bisher hat das Medikament für diese Applikation allerdings noch keine Zulassung in Deutschland; es kommt hier also im sog. Off-Label-Use zur Anwendung.

\section{Postiktale Phase}

Die postiktale Phase ist der Zustand nach einem epileptischen Krampfanfall. Typisch für diese Phase sind die Desorientierung und die Schläfrigkeit des Patienten. Um andere Gründe für das Krampfereignis auszuschließen, vervollständigt das Team jetzt die Untersuchungsbefunde, um mögliche Differenzialdiagnosen auszuschließen, z. B. eine Hypoglykämie oder ein Schädel-Hirn-Trauma.

\section{Forellenaugen und Zungenbiss}

Typische Hinweise auf ein zurückliegendes Krampfereignis sind neben lateraler oder bilateraler Verletzung der Zunge (Zungenbiss) auch petechiale Einblutungen im Bereich der Augen (Forellenaugen). Zudem sind eine Tachykardie und erhöhter Blutdruck typische postiktale Hinweise auf einen epileptischen Krampfanfall. Das Einnässen ist allerdings weniger spezifisch; auch bei einer Synkope können Patienten einnässen.

\section{Transport in die Klinik}

Jeder erstmals aufgetretene Krampfanfall muss in der Klinik differenzialdiagnostisch abgeklärt werden. Für einen bekannten Epileptiker ist es hingegen nicht zumutbar, bei jedem Krampfereignis in die Klinik gebracht zu werden. Dann sollte gemeinsam mit dem Patienten, wenn möglich unter Einbeziehung des behandelten Arztes, entschieden werden, ob eine Klinikeinweisung im Einzelfall notwendig ist. In dem oben beschriebenen Fall wird der Patient wegen der Kopfplatzwunde zur Wundversorgung in die Klinik gebracht.

\section{Prüfungsfragen}

\section{NOTFALLMEDIZIN}

Beschreiben Sie das Krankheitsbild Epilepsie. Welche Formen epileptischer Anfälle lassen sich grob unterscheiden? Auf welche Symptome achten Sie?

Unter Epilepsie versteht man ein Syndrom wiederkehrender, nicht provozierter Anfälle. Die Anfälle können in sehr unterschiedlicher Ausprägung vorkommen. Klinisch entscheidend für die Diagnose einer Epilepsie sind pathophysiologische exzessive hochsynchrone neuronale Entladungen, die sich im EEG darstellen lassen.

Es werden fokale von generalisierten Krampfanfällen unterschieden. Fokale Anfälle sind auf bestimmte Gehirnregionen beschränkt. Neben motorischen Störungen, z. B. Automatismen oder auf einzelne Körperregionen begrenzten klonischen Zuckungen und Spasmen, treten auch kognitive, sensorische oder emotionale Entgleisungen auf.

Bei den generalisierten Anfällen lassen sich Anfälle mit und Anfälle ohne motorische Störungen unterscheiden. Der sog. Grand-mal-Anfall ist wohl die bekannteste Form eines generalisierten Anfalls mit motorischen Störungen. Die Patienten beschreiben häufig im Vorfeld eines Krampfanfalls ein Vorgefühl (Schwindel, Unwohlsein, Sehstörungen ...), das als Aura bezeichnet wird. Danach folgt ein plötzlicher Sturz ohne Schutzreflexe, die Muskeln versteifen sich (tonische Phase) und es folgen nun Zuckungen der Arm-, Bein- und Nackenmuskulatur (klonische Phase). In dieser Phase ist es sehr wichtig, den Patienten vor weiteren Verletzungen zu schützen. Die letzte Phase des Anfalls ist geprägt von einer massiven Desorientierung und Schläfrigkeit (Nachschlafphase).

Außer diesen klassischen generalisierten Anfällen gibt es Petit-mal-Anfälle ohne motorische Störungen, die als Absencen auftreten. Bei einer Absence kommt zu einer kurzfristigen Bewusstseinspause. Der Patient hält kurz inne, bevor er in der Handlung fortfährt. In manchen Fällen wird die Absence von einem leisen Stöhnen begleitet. Dem Patienten ist die kurze Abwesenheit nicht bewusst.

\section{Mögliche weitere Fragen:}

- Der Krampfanfall ist ein Symptom, das bei einer Reihe von Notfällen auftritt. An welche Differenzialdiagnosen müssen Sie denken?

- Beschreiben Sie Streck- und/oder Beugesynergismen im Rahmen eines Schädel-Hirn-Traumas.

- Wie unterscheiden sich fokale und generalisierte Krampfereignisse? 
> Tab. 1 Kombination aus Einteilung nach Intelligenz, Schweregrad und sozialadaptivem Verhalten (Deutsche Gesellschaft für Kinder- und Jungendpsychiatrie und Psychotherapie u. a., 2007, S. 179 ff.), ergänzt um Soziale Anpassungen (Barhoff 1986 nach Wendeler 1993, S. 13 ff.) und psychologische Aspekte (Fröhlich/Haupt 1982, S. 21-22, ebd.).

\begin{tabular}{|c|c|c|c|}
\hline IQ-Wert nach ICD 10 & Schweregrad & Sozialadaptives Verhalten & Soziale Anpassungen \\
\hline leicht (50-69) & $\begin{array}{l}\text { leichte geistige } \\
\text { Behinderung }\end{array}$ & $\begin{array}{l}\text { " verzögerte Entwicklung } \\
\text { sozialer und kommunikativer } \\
\text { Fertigkeiten } \\
\text { " eigenständige Lebensfüh- } \\
\text { rung möglich }\end{array}$ & \multirow{2}{*}{$\begin{array}{l}\text { - besitzen alle grundlegenden Voraussetzungen } \\
\text { zur Selbstversorgung, werden aber nicht völlig } \\
\text { unabhängig } \\
\text { - Sprache genügt für Verständigungszwecke, ist } \\
\text { aber meist etwas umständlich } \\
\text { - können ansatzweise oder gar nicht lesen } \\
\text { - haben eingeschränkte Fähigkeiten im Umgang } \\
\text { mit Geld } \\
\text { - können einfache berufliche Tätigkeiten ausüben, } \\
\text { allerdings nicht auf dem freien Arbeitsmarkt } \\
\text { - sind zu beidgeschlechtlichen Freundschaften } \\
\text { fähig, können aber die Rolle als Ehepartner oder } \\
\text { Eltern nicht ausfüllen }\end{array}$} \\
\hline mittel (35-49) & $\begin{array}{l}\text { mäßige geistige } \\
\text { Behinderung }\end{array}$ & $\begin{array}{l}\text { - begrenzte sprachliche und } \\
\text { kommunikative Fertigkeiten } \\
\text { - benötigt Betreuung und } \\
\text { Begleitung }\end{array}$ & \\
\hline schwer (20-34) & $\begin{array}{l}\text { schwere geistige } \\
\text { Behinderung }\end{array}$ & $\begin{array}{l}\text { - stark verzögerte motorische } \\
\text { Entwicklung } \\
\text { " sehr eingeschränkte sprachli- } \\
\text { che und kommunikative } \\
\text { Fertigkeiten } \\
\text { - braucht Pflege und Betreuung }\end{array}$ & $\begin{array}{l}\text { - können sich teilweise selbst versorgen, brauchen } \\
\text { nur bei schwierigen Aufgaben persönliche Pflege } \\
\text { und Hygienehilfe } \\
\text { - können sprechen, jedoch meist schlecht artikuliert } \\
\text { und schwer verständlich } \\
\text { - können weder lesen noch schreiben oder mit Zahlen } \\
\text { umgehen } \\
\text { - können kleinere Arbeiten in Werkstätten überneh- } \\
\text { men (Fördergruppen) } \\
\text { - bauen Beziehungen zu Gleichaltrigen auf }\end{array}$ \\
\hline schwerst $(<20)$ & $\begin{array}{l}\text { schwerste geistige } \\
\text { Behinderung }\end{array}$ & $\begin{array}{l}\text { - schwerste Retardierung mit } \\
\text { minimalen Funktionen in } \\
\text { Wahrnehmung und Motorik } \\
\text { - intensive Pflege nötig }\end{array}$ & $\begin{array}{l}\text { - haben eine individuelle Pflegebedürftigkeit } \\
\text { - haben sehr eingeschränkte Verfügbarkeit auch } \\
\text { einfachster motorischer Muster } \\
\text { - } \text { Fähigkeit zur Fortbewegung kann wegen körperli- } \\
\text { cher oder sensorischer Probleme stark einge- } \\
\text { schränkt sein } \\
\text { - haben eine reduzierte Kommunikationsfähigkeit, } \\
\text { Mimik und Gestik durch muskuläre Defizite } \\
\text { rudimentär } \\
\text { - häufig ist die Funktion des Kauens und Schluckens } \\
\text { beeinträchtigt } \\
\text { - verstehen einfache Aufforderungen und Anwei- } \\
\text { sungen } \\
\text { - können bei ausreichender Übung in geschützter } \\
\text { Umgebung zu ihrer eigenen Versorgung beitragen } \\
\text { - erscheinen oft sozial sehr isoliert } \\
\text { - zeigen häufig stereotypes Verhalten }\end{array}$ \\
\hline
\end{tabular}

\section{KOMMUNIKATION}

? Definieren Sie den Begriff „Menschen mit geistiger Behinderung". Welche Einteilungen kennen Sie? Beschreiben Sie, worauf Sie bei der Kommunikation achten.

Sowohl bei der Begriffsdefinition als auch bei der Einteilung von Menschen mit einer geistigen Behinderung gibt es je nach Fachdisziplin unterschiedliche Herangehensweisen. Die WHO definiert eine geistige Behinderung als signifikant verringerte Fähigkeit, neue oder komplexe Informationen zu verstehen und neue Fähigkeiten zu erlernen bzw. anzuwenden. Daher fehlt dem Menschen die Möglichkeit, ein unabhängiges Leben zu führen.
Unabhängig von der rein IQ-bezogenen Einteilung geistiger Behinderungen hat sich eine Einteilung nach den Kompetenzperspektiven und dem individuellen Hilfebedarf (sozialadaptives Verhalten) in leicht, mittel, schwer und schwerste geistige Behinderung bewährt ( $\vee$ Tab. 1).

Die Kommunikation sollte an die individuellen Möglichkeiten des Menschen angepasst sein. Bei Erwachsenen muss beachtet werden, den Patienten nicht wie ein Kleinkind zu behandeln. Um Ängste durch die ungewohnte Umgebung und Situation zu reduzieren, hilft es, ruhig und mit angepasster Lautstärke zu sprechen. Einfache, kurze Sätze, Vermeiden von Nebensätzen und Fachbegrif- 
fen sowie deutliches, dem Patienten zugewandtes Sprechen helfen (nicht nur) Menschen mit geistiger Behinderung, das Gegenüber zu verstehen.

\section{Mögliche weitere Fragen:}

- Welche möglichen Ängste werden Alexander im oben beschriebenen Fall wohl beschäftigen? Reflektieren Sie seine möglichen Bedürfnisse.

\section{RAHMENBEDINGUNGEN}

? Wie bewerten Sie die intranasale Gabe von Midazolam über ein MAD als sog. Off-Label-Use durch einen Notfallsanitäter?

Medikamente müssen für den Einsatz in Deutschland durch das Bundesinstitut für Arzneimittel und Medizinprodukte (BfArM) zugelassen sein. Das BfArM ist eine selbstständige Bundesoberbehörde mit dem Schwerpunkt Zulassung von Fertigarzneimitteln.

Für manche Einsatzgebiete von Medikamenten gibt es keine offizielle Zulassung. Insbesondere betrifft dies Einsatzgebiete, bei denen die Hersteller die hohen Kosten für die Zulassung scheuen. Um trotzdem eine Anwendung zu ermöglichen, gibt es die Möglichkeit der Anwendung im Off-Label-Use. Konkret übernimmt dabei der behandelnde Arzt die Verantwortung für die sach- und fachgerechte Anwendung. Selbstverständlich wird vorausgesetzt, dass sich der Arzt vor einer Anwendung umfassend informiert hat und über die notwendigen Kompetenzen für die Anwendung und die Beherrschung eventueller Nebenwirkungen verfügt.

Beim Off-Label-Use handelt es sich immer um eine Einzelfallentscheidung. Diese Einzelfallentscheidungen im Rahmen eines Algorithmus für Notfallsanitäter freizugeben kann sehr kritisch gesehen werden. In anderen Algorithmen zur Behandlung von Krampfanfällen durch Notfallsanitäter wird deshalb die rektale oder intramuskuläre Gabe von Midazolam empfohlen.

\section{Mögliche weitere Fragen:}

- Alexander ist 21 Jahre alt und somit voll geschäftsfähig. Seine Betreuerin gibt an, er habe eine geistige Behinderung. Setzen Sie sich kritisch mit der besonderen Problematik für das Einsatzteam auseinander. Wie würden Sie vorgehen, wenn Alexander einen Transport in die Klinik ablehnt?

\section{KOMMENTAR}

von Frank Flake, 2. Vorsitzender Deutscher Berufsverband Rettungsdienst e. V. (DBRD)

Insbesondere bei einem aktiven Krampfanfall stellt das Legen eines peripher-venösen Zugangs das ein- gesetzte Personal vor eine besondere Herausforderung. Gerade in solchen Situationen ist die intranasale Applikation des notwendigen Medikaments eine hervorragende Option.

Bevor im Jahr 2005 das MAD-System eingeführt wurde, wurden Benzodiazepine vereinzelt mittels einer Spritze in die Nase appliziert. Leider wurde so selten der Bereich in der Nase erreicht, der eine schnelle Aufnahme des Wirkstoffs in das ZNS ermöglicht. Dazu müssen Medikamente in die sog. Regio olfactoria eingebracht werden. Von dort erreichen die Medikamente sehr schnell ihren Wirkort. Tim Wolfe, ein amerikanischer Notfallmediziner, hat das MAD-System zusammen mit einem Ingenieur entwickelt eine segensreiche Erfindung, die heute aus der Notfallmedizin nicht mehr wegzudenken ist.

Bei der intranasalen Applikation von Benzodiazepinen treten, bei korrekter Dosierung, weniger Nebenwirkungen auf. Ebenso haben Wissenschaftler festgestellt, dass das Wiederauftreten von Krampfanfällen nach der intranasalen Applikation seltener vorkommt. Die Nase des Menschen ist ein exponiertes Körperteil, das in den meisten Fällen gut erreicht werden kann - so auch bei einem aktiven Krampfanfall. Intranasal können aber nicht nur Benzodiazepine, sondern beispielsweise auch Analgetika appliziert werden. Gerade wenn sich Patienten in schwer zugänglichen Bereichen befinden, ist die intranasale Applikation ebenfalls eine hervorragende Applikationsform.

\section{Interessenkonflikt}

Die Autorinnen/Autoren geben an, dass kein Interessenkonflikt besteht.

\section{Autorinnen/Autoren}

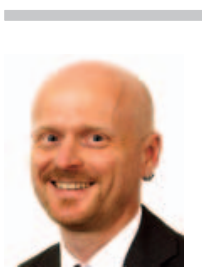

\section{Rico Kuhnke}

MA. Gesamtschulleiter der DRK-Landesschule Baden-Württemberg. Er war viele Jahre als Lehrrettungsassistent tätig und hat sein pädagogisches Masterstudium berufsbegleitend abgeschlossen. Er ist Notfallsanitäter und Mitherausgeber von retten!

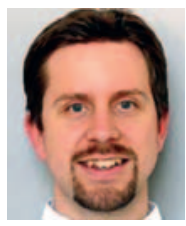

\section{Thomas Ahne}

Dr. med. Facharzt für Anästhesiologie mit Zusatzbezeichnung Notfallmedizin. Der Mitherausgeber von retten! macht derzeit einen Quereinstieg in die Allgemeinmedizin am Gesundheitszentrum Todtnau. Präklinisch ist er, boden- wie luftgebunden, in Deutschland, Österreich und der Schweiz tätig. 
Korrespondenzadresse

\section{Rico Kuhnke}

Schulleiter

Deutsches Rotes Kreuz

Landesschule Baden-Württemberg

Karl-Berner-Straße 6

72285 Pfalzgrafenweiler

r.kuhnke@drk-Is.de
Bibliografie

DOI https://doi.org/10.1055/a-1136-3774

retten 2020; 9: 238-245

(c) Georg Thieme Verlag KG, Stuttgart · New York

ISSN 2193-2387 